Commun. Fac. Sci. Univ. Ank. Ser. A1 Math. Stat.

Volume 68, Number 2, Pages 1939-1949(2019)

DOI: $10.31801 /$ cfsuasmas.000000

ISSN 1303-5991 E-ISSN 2618-6470

http://communications.science.ankara.edu.tr/index.php?series=A1

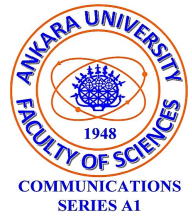

\title{
THE MATRIX SEQUENCE IN TERMS OF BI-PERIODIC FIBONACCI NUMBERS
}

\author{
ARZU COSKUN AND NECATI TASKARA
}

\begin{abstract}
In this paper, we define the bi-periodic Fibonacci matrix sequence that represent bi-periodic Fibonacci numbers. Then, we investigate generating function, Binet formula and summations of bi-periodic Fibonacci matrix sequence. After, we say that some behaviours of bi-periodic Fibonacci numbers can be obtained via the properties of this new matrix sequence. Finally, we express that well-known matrix sequences such as Fibonacci, Pell, $k$-Fibonacci matrix sequences are special cases of this generalized matrix sequence.
\end{abstract}

\section{Introduction And Preliminaries}

The special number sequences such as Fibonacci, Lucas, Pell, Jacobsthal and their properties have been investigated in many articles and books (see, for example [1, 3, 4, 6, 7, 9, 14, 16, 17] and the references cited therein). The Fibonacci numbers have attracted the attention of mathematicians because of their intrinsic theory and applications. The ratio of two consecutive of these numbers converges to the Golden section $\alpha=\frac{1+\sqrt{5}}{2}$. It is also clear that the ratio has so many applications in, specially, Physics, Engineering, Architecture, etc. 8].

After the study of Fibonacci numbers started in the beginning of 13 . century, many authors have generalized this sequence in different ways. One of those generalizations was published in 2009 by Edson et al. in 3. In this reference, the authors defined the bi-periodic Fibonacci $\left\{q_{n}\right\}_{n \in \mathbb{N}}$ sequence as

$$
q_{n}=\left\{\begin{array}{ll}
a q_{n-1}+q_{n-2}, & \text { if } n \text { is even } \\
b q_{n-1}+q_{n-2}, & \text { if } n \text { is odd }
\end{array},\right.
$$

where $q_{0}=0, q_{1}=1$ and $a, b$ are nonzero real numbers.

Also, in [11, it is defined a sequence $\left\{q_{m}\right\}_{m \in \mathbb{N}}$, for all $m \geq 2, q_{m}=a_{t} q_{m-1}+q_{m-2}$ with initial conditions $q_{0}=0, q_{1}=1$ where $m \equiv t(\bmod r)$ and $a_{0}, a_{1}, \ldots, a_{r-1}$

Received by the editors: May 01, 2018; Accepted: April 06, 2019.

2010 Mathematics Subject Classification. 11B39; 15A24.

Key words and phrases. Bi-periodic Fibonacci matrix sequence, bi-periodic Fibonacci numbers, Binet formula, generating function.

(C)2019 Ankara University Communications Faculty of Sciences University of Ankara-Series A1 Mathematics and Statistics 
positive numbers. For $r=2$, the author called the sequence $\left\{q_{m}\right\}$ as the generalized Fibonacci sequences and it is studied in 3. But, it remains open to find a closed form of the generating function for general $\left\{q_{m}\right\}$. In this paper, the author solve this open problem, that is, he find a closed form of the generating function for $\left\{q_{m}\right\}$ in terms of the continuant. In addition, in [10, this general conditional sequences are generalized as non-homogeneous conditional sequences and developed some techniques for the solution.

On the other hand, the matrix sequences have taken so much interest for different type of numbers $(2,15,12,13,15,18)$. In [5], the authors defined $(s, t)$-Pell and $(s, t)$-Pell-Lucas sequences and $(s, t)$-Pell and $(s, t)$-Pell-Lucas matrix sequences, also gave their some properties. Yazlik et al., in [18, establish generalized $(s, t)$ matrix sequences and present some important relationships among $(s, t)$-Fibonacci and $(s, t)$-Lucas sequences and their matrix sequences.

The goal of this paper is to define the related matrix sequence for bi-periodic Fibonacci numbers as the first time in the literature. Then, it will be given the generating function, Binet formula and summation formulas for this new generalized matrix sequence. Thus, some fundamental properties of bi-periodic Fibonacci numbers can be obtained by taking into account this generalized matrix sequence and its properties. By using the results in Sections 2, we have a great opportunity to obtain some new properties over this matrix sequence.

\section{The matrix RePresentation of BI-PERIOdiC FibonacCi numbers}

In this section, we mainly focus on the matrix sequence of bi-periodic Fibonacci numbers to get some important results. In fact, we also present the generating function, Binet formula and summations for the matrix sequence.

Hence, in the following, we firstly define the bi-periodic Fibonacci matrix sequence.

Definition 2.1. For $n \in \mathbb{N}$ and $a, b \in \mathbb{R}-\{0\}$, the bi-periodic Fibonacci matrix sequence $\mathcal{F}_{n}(a, b)$ is defined by

$$
\mathcal{F}_{n}(a, b)= \begin{cases}a \mathcal{F}_{n-1}(a, b)+\mathcal{F}_{n-2}(a, b), & n \text { even } \\ b \mathcal{F}_{n-1}(a, b)+\mathcal{F}_{n-2}(a, b), & n \text { odd }\end{cases}
$$

with initial conditions $\mathcal{F}_{0}(a, b)=\left(\begin{array}{cc}1 & 0 \\ 0 & 1\end{array}\right), \mathcal{F}_{1}(a, b)=\left(\begin{array}{cc}b & \frac{b}{a} \\ 1 & 0\end{array}\right)$.

In Definition 2.1, the matrix $\mathcal{F}_{1}$ is analogue to the Fibonacci $Q$-matrix which exists for Fibonacci numbers.

In the following theorem, we give the $n$th general term of the matrix sequence in (2.1) via bi-periodic Fibonacci numbers.

Theorem 2.2. For any integer $n \geq 0$, we have the matrix sequence

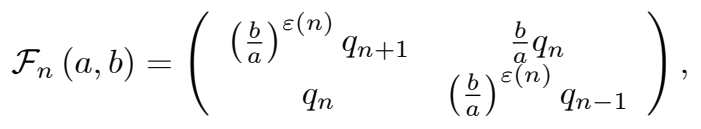


where $\varepsilon(n)=n-2\left\lfloor\frac{n}{2}\right\rfloor$.

Proof. We know that $q_{2}=a, q_{-1}=q_{1}=1$ and $q_{0}=0$. So, the first and second steps of the induction is obtained as follows:

$$
\begin{aligned}
& \mathcal{F}_{0}(a, b)=\left(\begin{array}{ll}
1 & 0 \\
0 & 1
\end{array}\right)=\left(\begin{array}{cc}
q_{1} & \frac{b}{a} q_{0} \\
q_{0} & q_{-1}
\end{array}\right), \\
& \mathcal{F}_{1}(a, b)=\left(\begin{array}{cc}
b & \frac{b}{a} \\
1 & 0
\end{array}\right)=\left(\begin{array}{cc}
\frac{b}{a} q_{2} & \frac{b}{a} q_{1} \\
q_{1} & \frac{b}{a} q_{0}
\end{array}\right) .
\end{aligned}
$$

Actually, by assuming the equation in 2.2 holds for all $n=k \in \mathbb{Z}^{+}$, we can end up the proof if we manage to show that the case also holds for $n=k+1$ :

$$
\begin{aligned}
& \mathcal{F}_{k+1}(a, b)=a^{\varepsilon(k)} b^{1-\varepsilon(k)} \mathcal{F}_{k}(a, b)+\mathcal{F}_{k-1}(a, b)
\end{aligned}
$$

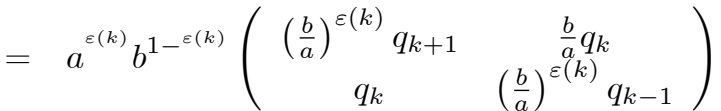

$$
\begin{aligned}
& +\left(\begin{array}{ccc}
\left(\frac{b}{a}\right)^{\varepsilon(k-1)} & & \frac{b}{a} q_{k-1} \\
& q_{k-1} & \left(\frac{b}{a}\right)^{\varepsilon(k-1)} q_{k-2}
\end{array}\right) \\
& =\left\{\begin{array}{c}
\left(\begin{array}{cc}
\frac{b}{a} q_{k+2} & \frac{b}{a} q_{k+1} \\
q_{k+1} & \frac{b}{a} q_{k}
\end{array}\right), k \text { even } \\
\left(\begin{array}{cc}
q_{k+2} & \frac{b}{a} q_{k+1} \\
q_{k+1} & q_{k}
\end{array}\right), k \text { odd }
\end{array} .\right.
\end{aligned}
$$

By combining this partial function, we obtain

$$
\mathcal{F}_{k+1}(a, b)=\left(\begin{array}{ccc}
\left(\frac{b}{a}\right)^{\varepsilon(k+1)} & q_{k+2} & \frac{b}{a} q_{k+1} \\
& q_{k+1} & \left(\frac{b}{a}\right)^{\varepsilon(k+1)} q_{k}
\end{array}\right) .
$$

Theorem 2.3. Let $\mathcal{F}_{n}(a, b)$ be as in 2.2.). Then the following equality is valid for all positive integers:

$$
\operatorname{det}\left(\mathcal{F}_{n}(a, b)\right)=\left(-\frac{b}{a}\right)^{\varepsilon(n)}
$$

Proof. By using the induction on $n$, we get

$$
\operatorname{det}\left(\mathcal{F}_{n}(a, b)\right)=\left\{\begin{array}{c}
-\frac{b}{a}, n \text { odd } \\
1, n \text { even }
\end{array}\right.
$$

which is desired.

In [3, the authors obtained the Cassini identity for bi-periodic Fibonacci numbers. With a different approach, so as a consequence of Theorem 2.2 and Theorem 2.3 , we can obtain this identity. In fact, in the following corollary, we just compare the determinants. 
Corollary 2.4. Cassini identity for bi-periodic Fibonacci sequence can also be obtained using bi-periodic Fibonacci matrix sequence. That is, by using Theorem 2.2 and Theorem 2.3, we can write

$$
\left(\frac{b}{a}\right)^{2 \varepsilon(n)} q_{n+1} q_{n-1}-\frac{b}{a} q_{n}^{2}=\left(-\frac{b}{a}\right)^{\varepsilon(n)} .
$$

Thus, we obtain

$$
a^{1-\varepsilon(n)} b^{\varepsilon(n)} q_{n+1} q_{n-1}-a^{\varepsilon(n)} b^{1-\varepsilon(n)} q_{n}^{2}=a(-1)^{n} .
$$

Theorem 2.5. For bi-periodic Fibonacci matrix sequence, we have the generating function

$$
\sum_{i=0}^{\infty} \mathcal{F}_{i}(a, b) x^{i}=\frac{1}{1-(a b+2) x^{2}+x^{4}}\left(\begin{array}{cc}
1+b x-x^{2} & \frac{b}{a} x+b x^{2}-\frac{b}{a} x^{3} \\
x+a x^{2}-x^{3} & 1-(a b+1) x^{2}+b x^{3}
\end{array}\right) .
$$

Proof. Assume that $G(x)$ is the generating function for the sequence $\left\{\mathcal{F}_{n}\right\}_{n \in \mathbb{N}}$. Then, we have

$$
G(x)=\sum_{i=0}^{\infty} \mathcal{F}_{i}(a, b) x^{i}=\mathcal{F}_{0}(a, b)+\mathcal{F}_{1}(a, b) x+\sum_{i=2}^{\infty} \mathcal{F}_{i}(a, b) x^{i} .
$$

Note that

$$
-b x G(x)=-b x \sum_{i=0}^{\infty} \mathcal{F}_{i}(a, b) x^{i}=-b x \mathcal{F}_{0}(a, b)-b \sum_{i=2}^{\infty} \mathcal{F}_{i-1}(a, b) x^{i}
$$

and

$$
-x^{2} G(x)=-\sum_{i=2}^{\infty} \mathcal{F}_{i-2}(a, b) x^{i} .
$$

Thus, we can write

$$
\begin{aligned}
\left(1-b x-x^{2}\right) G(x)= & \mathcal{F}_{0}(a, b)+x\left(\mathcal{F}_{1}(a, b)-b \mathcal{F}_{0}(a, b)\right) \\
& +\sum_{i=2}^{\infty}\left(\mathcal{F}_{i}(a, b)-b \mathcal{F}_{i-1}(a, b)-\mathcal{F}_{i-2}(a, b)\right) x^{i}
\end{aligned}
$$

Since $\mathcal{F}_{2 i+1}(a, b)=b \mathcal{F}_{2 i}(a, b)+\mathcal{F}_{2 i-1}(a, b)$, we get

$$
\begin{aligned}
\left(1-b x-x^{2}\right) G(x)= & \mathcal{F}_{0}(a, b)+x\left(\mathcal{F}_{1}(a, b)-b \mathcal{F}_{0}(a, b)\right) \\
& +\sum_{i=1}^{\infty}\left(\mathcal{F}_{2 i}(a, b)-b \mathcal{F}_{2 i-1}(a, b)-\mathcal{F}_{2 i-2}(a, b)\right) x^{2 i} \\
= & \mathcal{F}_{0}(a, b)+x\left(\mathcal{F}_{1}(a, b)-b \mathcal{F}_{0}(a, b)\right) \\
& +(a-b) x \sum_{i=1}^{\infty} \mathcal{F}_{2 i-1}(a, b) x^{2 i-1} .
\end{aligned}
$$


Now, let

$$
g(x)=\sum_{i=1}^{\infty} \mathcal{F}_{2 i-1}(a, b) x^{2 i-1} .
$$

Since

$$
\begin{aligned}
\mathcal{F}_{2 i+1}(a, b) & =b \mathcal{F}_{2 i}(a, b)+\mathcal{F}_{2 i-1}(a, b) \\
& =b\left(a \mathcal{F}_{2 i-1}(a, b)+\mathcal{F}_{2 i-2}(a, b)\right)+\mathcal{F}_{2 i-1}(a, b) \\
& =(a b+1) \mathcal{F}_{2 i-1}(a, b)+b \mathcal{F}_{2 i-2}(a, b) \\
& =(a b+1) \mathcal{F}_{2 i-1}(a, b)+\mathcal{F}_{2 i-1}(a, b)-\mathcal{F}_{2 i-3}(a, b) \\
& =(a b+2) \mathcal{F}_{2 i-1}(a, b)-\mathcal{F}_{2 i-3}(a, b),
\end{aligned}
$$

we have

$$
\begin{aligned}
\left(1-(a b+2) x^{2}+x^{4}\right) g(x)= & \mathcal{F}_{1}(a, b) x+\mathcal{F}_{3}(a, b) x^{3}-(a b+2) \mathcal{F}_{1}(a, b) x^{3} \\
& +\sum_{i=3}^{\infty}\left(\begin{array}{c}
\mathcal{F}_{2 i-1}(a, b)-(a b+2) \mathcal{F}_{2 i-3}(a, b) \\
+\mathcal{F}_{2 i-5}(a, b)
\end{array}\right) x^{2 i-1}
\end{aligned}
$$

Therefore,

$$
\begin{aligned}
g(x) & =\frac{\mathcal{F}_{1}(a, b) x+\mathcal{F}_{3}(a, b) x^{3}-(a b+2) \mathcal{F}_{1}(a, b) x^{3}}{1-(a b+2) x^{2}+x^{4}} \\
& =\frac{\mathcal{F}_{1}(a, b) x+\left(b \mathcal{F}_{0}(a, b)-\mathcal{F}_{1}(a, b)\right) x^{3}}{1-(a b+2) x^{2}+x^{4}}
\end{aligned}
$$

and as a result, we get

$$
G(x)=\frac{\begin{array}{c}
\mathcal{F}_{0}(a, b)+x \mathcal{F}_{1}(a, b)+x^{2}\left(a \mathcal{F}_{1}(a, b)-\mathcal{F}_{0}(a, b)-a b \mathcal{F}_{0}(a, b)\right) \\
+x^{3}\left(b \mathcal{F}_{0}(a, b)-\mathcal{F}_{1}(a, b)\right)
\end{array}}{1-(a b+2) x^{2}+x^{4}} .
$$

which is desired equality.

Theorem 2.6. For every $n \in \mathbb{N}$, we write the Binet formula for the bi-periodic Fibonacci matrix sequence as the form

$$
\mathcal{F}_{n}(a, b)=A_{1}(n)\left(\alpha^{n}-\beta^{n}\right)+B_{1}(n)\left(\alpha^{2\left\lfloor\frac{n}{2}\right\rfloor+2}-\beta^{2\left\lfloor\frac{n}{2}\right\rfloor+2}\right),
$$

where

$$
\begin{aligned}
& A_{1}(n)=\frac{\left[\mathcal{F}_{1}(a, b)-b \mathcal{F}_{0}(a, b)\right]^{\varepsilon(n)}\left[a \mathcal{F}_{1}(a, b)-\mathcal{F}_{0}(a, b)-a b \mathcal{F}_{0}(a, b)\right]^{1-\varepsilon(n)}}{(a b)^{\left\lfloor\frac{n}{2}\right\rfloor}(\alpha-\beta)}, \\
& B_{1}(n)=\frac{b^{\varepsilon(n)} \mathcal{F}_{0}(a, b)}{(a b)^{\left\lfloor\frac{n}{2}\right\rfloor+1}(\alpha-\beta)},
\end{aligned}
$$

such that $\alpha=\frac{a b+\sqrt{a^{2} b^{2}+4 a b}}{2}, \beta=\frac{a b-\sqrt{a^{2} b^{2}+4 a b}}{2}$ and $\varepsilon(n)=n-2\left\lfloor\frac{n}{2}\right\rfloor$. 
Proof. We know that the generating function of bi-periodic Fibonacci matrix sequence is

$$
G(x)=\frac{\begin{array}{c}
\mathcal{F}_{0}(a, b)+x \mathcal{F}_{1}(a, b)+x^{2}\left(a \mathcal{F}_{1}(a, b)-\mathcal{F}_{0}(a, b)-a b \mathcal{F}_{0}(a, b)\right) \\
+x^{3}\left(b \mathcal{F}_{0}(a, b)-\mathcal{F}_{1}(a, b)\right)
\end{array}}{1-(a b+2) x^{2}+x^{4}} .
$$

Using the partial fraction decomposition, we rewrite $G(x)$ as

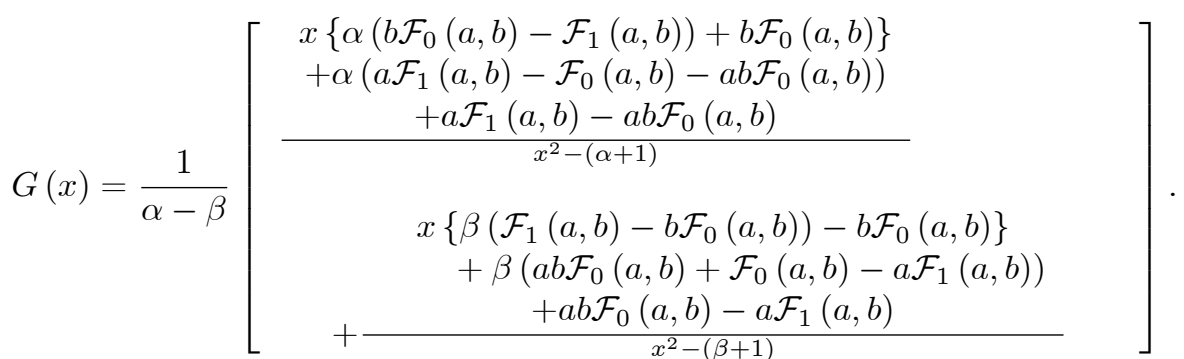

Since the Maclaurin series expansion of the function $\frac{A-B x}{x^{2}-C}$ is given by

$$
\frac{A-B x}{x^{2}-C}=\sum_{n=0}^{\infty} B C^{-n-1} x^{2 n+1}-\sum_{n=0}^{\infty} A C^{-n-1} x^{2 n},
$$

the generating function $G(x)$ can be expressed as

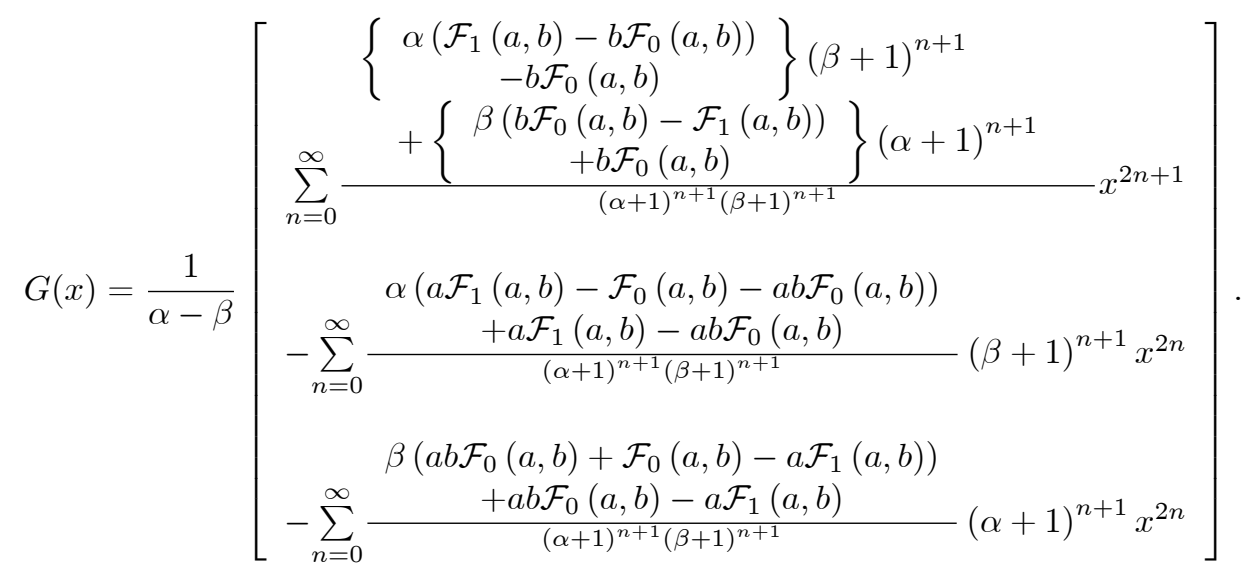

By using properties of $\alpha$ and $\beta$, since we know that $\alpha$ and $\beta$ are roots of equation $X^{2}-a b X-a b=0$, we obtain

$$
G(x)=\frac{1}{\alpha-\beta} \sum_{n=0}^{\infty}\left(\frac{1}{a b}\right)^{n+1}\left(\begin{array}{c}
-a b\left(\mathcal{F}_{1}(a, b)-b \mathcal{F}_{0}(a, b)\right) \beta^{2 n+1} \\
-a b\left(b \mathcal{F}_{0}(a, b)-\mathcal{F}_{1}(a, b)\right) \alpha^{2 n+1} \\
-b \mathcal{F}_{0}(a, b) \beta^{2 n+2}+b \mathcal{F}_{0}(a, b) \alpha^{2 n+2}
\end{array}\right) x^{2 n+1}
$$


THE MATRIX SEQUENCE IN TERMS OF BI-PERIODIC FIBONACCI NUMBERS 1945

$$
\begin{aligned}
+ & \frac{1}{\alpha-\beta} \sum_{n=0}^{\infty}\left(\frac{1}{a b}\right)^{n+1}\left(\begin{array}{c}
-a b\left(\begin{array}{c}
a \mathcal{F}_{1}(a, b)-\mathcal{F}_{0}(a, b) \\
-a b \mathcal{F}_{0}(a, b) \\
-a b\left(\begin{array}{c}
a b \mathcal{F}_{0}(a, b)+\mathcal{F}_{0}(a, b) \\
-a \mathcal{F}_{1}(a, b)
\end{array}\right) \beta^{2 n} \\
-
\end{array}\right) \alpha^{2 n} \\
-\mathcal{F}_{0}(a, b) \beta^{2 n+2}+\mathcal{F}_{0}(a, b) \alpha^{2 n+2}
\end{array}\right) x^{2 n} . \\
G(x)= & \sum_{n=0}^{\infty}\left(\frac{1}{a b}\right)^{n} \frac{\left(\mathcal{F}_{1}(a, b)-b \mathcal{F}_{0}(a, b)\right)\left(\alpha^{2 n+1}-\beta^{2 n+1}\right)}{\alpha-\beta} x^{2 n+1} \\
& +\sum_{n=0}^{\infty}\left(\frac{1}{a b}\right)^{n+1} \frac{b \mathcal{F}_{0}(a, b)\left(\alpha^{2 n+2}-\beta^{2 n+2}\right)}{\alpha-\beta} x^{2 n+1} \\
& +\sum_{n=0}\left(\frac{1}{a b}\right)^{n} \frac{\left(a b \mathcal{F}_{0}(a, b)+\mathcal{F}_{0}(a, b)-a \mathcal{F}_{1}(a, b)\right)\left(\beta^{2 n}-\alpha^{2 n}\right)}{\alpha-\beta} x^{2 n} \\
& +\sum_{n=0}^{\infty}\left(\frac{1}{a b}\right)^{n+1} \frac{\mathcal{F}_{0}(a, b)\left(\alpha^{2 n+2}-\beta^{2 n+2}\right)}{\alpha-\beta} x^{2 n} .
\end{aligned}
$$

Combining the sums, we get

$$
\begin{aligned}
G(x)=\sum_{n=0}^{\infty}\{- & {\left[\mathcal{F}_{1}(a, b)-b \mathcal{F}_{0}(a, b)\right]^{\varepsilon(n)}\left[a \mathcal{F}_{1}(a, b)-\mathcal{F}_{0}(a, b)-a b \mathcal{F}_{0}(a, b)\right]^{1-\varepsilon(n)} } \\
& \left.\times\left(\frac{\alpha^{n}-\beta^{n}}{(a b)^{\left\lfloor\frac{n}{2}\right\rfloor}(\alpha-\beta)}\right)+b^{\varepsilon(n)} \mathcal{F}_{0}(a, b)\left(\frac{\alpha^{2\left(\left\lfloor\frac{n}{2}\right\rfloor+1\right)}-\beta^{2\left(\left\lfloor\frac{n}{2}\right\rfloor+1\right)}}{(a b)^{\left\lfloor\frac{n}{2}\right\rfloor+1}(\alpha-\beta)}\right)\right\} x^{n} .
\end{aligned}
$$

Therefore, for all $n \geq 0$, from the definition of generating function, we have

$$
\mathcal{F}_{n}(a, b)=A_{1}(n)\left(\alpha^{n}-\beta^{n}\right)+B_{1}(n)\left(\alpha^{2\left\lfloor\frac{n}{2}\right\rfloor+2}-\beta^{2\left\lfloor\frac{n}{2}\right\rfloor+2}\right)
$$

which is desired.

Now, for bi-periodic Fibonacci matrix sequence, we give the some summations by considering the Binet formula.

Theorem 2.7. For $n \geq 1$, the following statements are true:

(i)

$$
\sum_{k=0}^{n-1} \mathcal{F}_{k}(a, b)=\frac{\begin{array}{c}
a^{\varepsilon(n)} b^{1-\varepsilon(n)} \mathcal{F}_{n}(a, b)+a^{1-\varepsilon(n)} b^{\varepsilon(n)} \mathcal{F}_{n-1}(a, b) \\
-a \mathcal{F}_{1}(a, b)+a b \mathcal{F}_{0}(a, b)-b \mathcal{F}_{0}(a, b)
\end{array}}{a b}
$$


(ii)

$$
\sum_{k=0}^{n} \mathcal{F}_{k}(a, b) x^{-k}=\frac{1}{1-(a b+2) x^{2}+x^{4}}\left\{\begin{array}{c}
\frac{\mathcal{F}_{n-1}(a, b)}{x^{n-1}}-\frac{\mathcal{F}_{n+1}(a, b)}{x^{n-3}} \\
+\frac{\mathcal{F}_{n}(a, b)}{x^{n}}-\frac{\mathcal{F}_{n+2}(a, b)}{x^{n-2}} \\
+x^{4} \mathcal{F}_{0}(a, b)+x^{3} \mathcal{F}_{1}(a, b) \\
-x^{2}\left[(a b+1) \mathcal{F}_{0}(a, b)-a \mathcal{F}_{1}(a, b)\right] \\
-x\left(\mathcal{F}_{1}(a, b)-b \mathcal{F}_{0}(a, b)\right)
\end{array}\right\} .
$$

where $\varepsilon(n)=n-2\left\lfloor\frac{n}{2}\right\rfloor$.

Proof. We omit the proof of (ii), because it can be done similarly as in the proof of (i). We investigate the situation according to the $n$ is even or odd. Thus, for even $n$

$$
\begin{aligned}
\sum_{k=0}^{n-1} \mathcal{F}_{k}(a, b)= & \sum_{k=0}^{\frac{n-2}{2}} \mathcal{F}_{2 k}(a, b)+\sum_{k=0}^{\frac{n-2}{2}} \mathcal{F}_{2 k+1}(a, b) \\
\sum_{k=0}^{n-1} \mathcal{F}_{k}(a, b)= & \sum_{k=0}^{\frac{n-2}{2}} \frac{a \mathcal{F}_{1}(a, b)-\mathcal{F}_{0}(a, b)-a b \mathcal{F}_{0}(a, b)}{(a b)^{k}} \frac{\alpha^{2 k}-\beta^{2 k}}{\alpha-\beta} \\
& +\sum_{k=0}^{\frac{n-2}{2}} \frac{\mathcal{F}_{0}(a, b)}{(a b)^{k+1}} \frac{\alpha^{2 k+2}-\beta^{2 k+2}}{\alpha-\beta} \\
& +\sum_{k=0}^{\frac{n-2}{2}} \frac{\mathcal{F}_{1}(a, b)-b \mathcal{F}_{0}(a, b)}{(a b)^{k}} \frac{\alpha^{2 k+1}-\beta^{2 k+1}}{\alpha-\beta} \\
& +\sum_{k=0}^{\frac{n-2}{2}} \frac{b \mathcal{F}_{0}(a, b)}{(a b)^{k+1}} \frac{\alpha^{2 k+2}-\beta^{2 k+2}}{\alpha-\beta}
\end{aligned}
$$

In here, simplifying the last equality, we imply

$$
\begin{aligned}
\sum_{k=0}^{n-1} \mathcal{F}_{k}(a, b)= & \frac{a \mathcal{F}_{1}(a, b)-\mathcal{F}_{0}(a, b)}{\alpha-\beta}\left\{\begin{array}{c}
\frac{\alpha^{n}-(a b)^{\frac{n}{2}}}{(a b)^{\frac{n}{2}-1}\left(\alpha^{2}-a b\right)} \\
-\frac{\beta^{n}-(a b) \frac{n}{2}^{\frac{n}{2}}}{(a b)^{\frac{n}{2}-1}\left(\beta^{2}-a b\right)}
\end{array}\right\} \\
& +\frac{\mathcal{F}_{0}(a, b)}{\alpha-\beta}\left\{\begin{array}{c}
\frac{\alpha^{n+2}-\alpha^{2}(a b)^{\frac{n}{2}}}{(a b)^{\frac{n}{2}}\left(\alpha^{2}-a b\right)} \\
-\frac{\beta^{n+2}-\beta^{2}(a b)^{\frac{n}{2}}}{(a b)^{\frac{n}{2}}\left(\beta^{2}-a b\right)}
\end{array}\right\} \\
& +\frac{\mathcal{F}_{1}(a, b)-b \mathcal{F}_{0}(a, b)}{\alpha-\beta}\left\{\begin{array}{c}
\frac{\alpha^{n+1}-\alpha(a b)^{\frac{n}{2}}}{(a b)^{\frac{n}{2}-1}\left(\alpha^{2}-a b\right)} \\
-\frac{\beta^{n+1}-\beta(a b)^{\frac{n}{2}}}{(a b)^{\frac{n}{2}-1}\left(\beta^{2}-a b\right)}
\end{array}\right\}
\end{aligned}
$$




$$
\begin{aligned}
& +\frac{b \mathcal{F}_{0}(a, b)}{\alpha-\beta}\left\{\begin{array}{c}
\frac{\alpha^{n+2}-\alpha^{2}(a b)^{\frac{n}{2}}}{(a b)^{\frac{n}{2}}\left(\alpha^{2}-a b\right)} \\
-\frac{\beta^{n+2}-\beta^{2}(a b)^{\frac{n}{2}}}{(a b)^{\frac{n}{2}}\left(\beta^{2}-a b\right)}
\end{array}\right\} . \\
\sum_{k=0}^{n-1} \mathcal{F}_{k}(a, b)= & \frac{a \mathcal{F}_{1}(a, b)-\mathcal{F}_{0}(a, b)-a b \mathcal{F}_{0}(a, b)}{\alpha-\beta}\left\{\begin{array}{c}
-\frac{\alpha^{n-2}-\beta^{n-2}}{(a b)^{\frac{n}{2}}} \\
+\frac{\alpha^{n}-\beta^{n}}{(a b)^{\frac{n}{2}+1}} \\
-\frac{\alpha^{2}-\beta^{2}}{(a b)^{2}}
\end{array}\right\} \\
& +\frac{\mathcal{F}_{0}(a, b)}{\alpha-\beta}\left\{-\frac{\alpha^{n}-\beta^{n}}{(a b)^{\frac{n}{2}+1}}+\frac{\alpha^{n+2}-\beta^{n+2}}{(a b)^{\frac{n}{2}+2}}-\frac{\alpha^{2}-\beta^{2}}{(a b)^{2}}\right\} \\
& +\frac{\mathcal{F}_{1}(a, b)-b \mathcal{F}_{0}(a, b)}{\alpha-\beta}\left\{-\frac{\alpha^{n-1}-\beta^{n-1}}{(a b)^{\frac{n}{2}}}+\frac{\alpha^{n+1}-\beta^{n+1}}{(a b)^{\frac{n}{2}+1}}\right\} \\
& +\frac{b \mathcal{F}_{0}(a, b)}{\alpha-\beta}\left\{-\frac{\alpha^{n}-\beta^{n}}{(a b)^{\frac{n}{2}+1}}+\frac{\alpha^{n+2}-\beta^{n+2}}{(a b)^{\frac{n}{2}+2}}-\frac{\alpha^{2}-\beta^{2}}{(a b)^{2}}\right\}
\end{aligned}
$$

and thus we get

$$
\begin{aligned}
& \sum_{k=0}^{n-1} \mathcal{F}_{k}(a, b)=\frac{\begin{array}{c}
\mathcal{F}_{n+1}(a, b)+\mathcal{F}_{n}(a, b)-\mathcal{F}_{n-1}(a, b)-\mathcal{F}_{n-2}(a, b) \\
-a \mathcal{F}_{1}(a, b)+a b \mathcal{F}_{0}(a, b)-b \mathcal{F}_{0}(a, b)
\end{array}}{a b} \\
& =\frac{b \mathcal{F}_{n}(a, b)+a \mathcal{F}_{n-1}(a, b)-a \mathcal{F}_{1}(a, b)+a b \mathcal{F}_{0}(a, b)-b \mathcal{F}_{0}(a, b)}{a b} .
\end{aligned}
$$

Similarly, for odd $n$, we obtain

$$
\begin{aligned}
\sum_{k=0}^{n-1} \mathcal{F}_{k}(a, b) & =\sum_{k=0}^{\frac{n-1}{2}} \mathcal{F}_{2 k}(a, b)+\sum_{k=0}^{\frac{n-3}{2}} \mathcal{F}_{2 k+1}(a, b) \\
& =\frac{a \mathcal{F}_{n}(a, b)+b \mathcal{F}_{n-1}(a, b)-a \mathcal{F}_{1}(a, b)+a b \mathcal{F}_{0}(a, b)-b \mathcal{F}_{0}(a, b)}{a b}
\end{aligned}
$$

As a result, we find

$$
\sum_{k=0}^{n-1} \mathcal{F}_{k}(a, b)=\frac{\begin{array}{c}
a^{\varepsilon(n)} b^{1-\varepsilon(n)} \mathcal{F}_{n}(a, b)+a^{1-\varepsilon(n)} b^{\varepsilon(n)} \mathcal{F}_{n-1}(a, b) \\
-a \mathcal{F}_{1}(a, b)+a b \mathcal{F}_{0}(a, b)-b \mathcal{F}_{0}(a, b)
\end{array}}{a b} .
$$

This completes the proof.

It is clear that the following result is correct for the bi-periodic Fibonacci matrix sequence as a consequence of the condition (ii) of Theorem 2.7 
Corollary 2.8. For $k>0$, we have

$$
\sum_{k=0}^{\infty} \mathcal{F}_{k}(a, b) x^{-k}=\frac{x}{1-(a b+2) x^{2}+x^{4}}\left[\begin{array}{cc}
x^{3}+b x^{2}-x & \frac{b}{a} x^{2}+b x-\frac{b}{a} \\
x^{2}+a x-1 & x^{3}-(a b+1) x+b
\end{array}\right] .
$$

\section{CONCLUSion}

In this paper, we define bi-periodic matrix sequence and give some properties of this new sequence. Thus, it is obtained a new genaralization for the matrix sequences and number sequences that have the similar recurrence relation in the literature. By taking into account this generalized matrix sequence and its properties, it also can be obtained properties of bi-periodic Fibonacci numbers. That is, if we compare the $2 n d$ row and $1 s t$ column entries of obtained equalities for matrix sequence in Section 2, we can get some properties for bi-periodic Fibonacci numbers. Also, some well-known matrix sequences, such as Fibonacci, Pell and $k$ Fibonacci are special cases of $\left\{\mathcal{F}_{n}(a, b)\right\}$ matrix sequence. That is, if we choose the different values of $a$ and $b$, then we obtain the summations, generating functions, Binet formulas of the well-known matrix sequence in the literature:

- If we replace $a=b=1$ in $\mathcal{F}_{n}(a, b)$, we obtain the generating function, Binet formula and summations for Fibonacci matrix sequence and Fibonacci numbers.

- If we replace $a=b=2$ in $\mathcal{F}_{n}(a, b)$, we obtain the generating function, Binet formula and summations for Pell matrix sequence and Pell numbers.

- If we replace $a=b=k$ in $\mathcal{F}_{n}(a, b)$, we obtain the generating function, Binet formula and summations for $k$-Fibonacci matrix sequence and $k$-Fibonacci numbers.

\section{REFERENCES}

[1] Bilgici G., Two generalizations of Lucas sequence, Applied Mathematics and Computation, 245 (2014), 526-538.

[2] Civciv H., Türkmen R., On the $(s, t)$-Fibonacci and Fibonacci matrix sequences, Ars Combinatoria, 87 (2008), 161-173.

[3] Edson M., Yayanie O., A new Generalization of Fibonacci sequence and Extended Binet's Formula, Integers, 9 (2009), 639-654.

[4] Falcon S., Plaza A., On the Fibonacci $k$-numbers, Chaos, Solitons $\& 5$ Fractal, 32 (2007), 1615-1624.

[5] Gulec H.H., Taskara N., On the $(s, t)$-Pell and $(s, t)$-Pell-Lucas sequences and their matrix representations, Applied Mathematics Letter, 25 (2012), 1554-1559.

[6] Horadam A.F., A generalized Fibonacci sequence, Math. Mag., 68 (1961), 455-459.

[7] Koshy T., Fibonacci and Lucas Numbers with Applications, John Wiley and Sons Inc, NY, 2001.

[8] Marek-Crnjac L, The mass spectrum of high energy elementary particles via El Naschie's golden mean nested oscillators, the Dunkerly-Southwell eigenvalue theorems and KAM, Chaos, Solutions \& Fractals, 18(1) (2003), 125-133.

[9] Ocal A.A., Tuglu N., Altinisik E., On the representation of $k$-generalized Fibonacci and Lucas numbers, Applied Mathematics and Computations, 170 (1) (2005), 584-596. 
[10] Panario D., Sahin M., Wang Q., Non-homogeneous conditional recurrences, Linear and Multilinear Algebra, 66 (10) (2018), 2089-2099.

[11] Sahin M., The generating function of a family of the sequences in terms of the continuant, Applied Mathematics and Computations, 217 (12) (2011), 5416-5420.

[12] Tan E., Ekin A.B., Some identities on conditional sequences by using matrix method, Miskolc Mathematical Notes, 18 (1) (2017), 469-477.

[13] Tan E., On bi-periodic Fibonacci and Lucas numbers by matrix method, Ars Combinatoria, 133 (2017), 107-113.

[14] Tasci D., Firengiz M.C., Incomplete Fibonacci and Lucas p-numbers, Mathematical and Computer Modelling, 52(9) (2010), 1763-1770.

[15] Uslu K., Uygun S., The $(s, t)$ Jacobsthal and $(s, t)$ Jacobsthal-Lucas Matrix Sequences, Ars Combinatoria, 108 (2013), 13-22.

[16] Vajda S., Fibonacci \& Lucas numbers and the golden section. Theory and Applications, Ellis Horwood Limited, 1989.

[17] Yazlik Y., Taskara N., A note on generalized $k$-Horadam sequence, Computers and Mathematics with Applications, 63 (2012), 36-41.

[18] Yazlik Y., Taskara N., Uslu K., Yilmaz N., The Generalized $(s, t)$-Sequence and its Matrix Sequence, American Institute of Physics (AIP) Conf. Proc., 1389 (2012), 381-384.

Current address: Arzu Coskun: Department of Mathematics, Faculty of Science, Selcuk University, Campus, 42075, Konya - Turkey

E-mail address: arzucoskun58@gmail.com

ORCID Address: http://orcid.org/0000-0002-7755-5747

E-mail address: taskara@selcuk.edu.tr

Current address: Necati Taskara: Department of Mathematics, Faculty of Science, Selcuk University, Campus, 42075, Konya - Turkey

ORCID Address: http://orcid.org/0000-0001-7974-435X 